\title{
Study of Serum Uric Acid Level in Hypertension
}

\author{
Ankit Vakil ${ }^{1}$, Pradip Vrkariya ${ }^{2}$, Viral Barafiwala ${ }^{3}$ Komal Gamit $^{4}$ Dharmil \\ Patel $^{5}$, Nilesh Doctor ${ }^{6}$ \\ 1,2,3,4,5 Resident doctor, Department of Internal Medicine, SMIMER, Surat \\ ${ }^{6}$ Associatet professor,Department of Internal Medicine, SMIMER, Surat
}

\begin{abstract}
:
Introduction: HTN is a sustained elevation of systemic arterial pressure. Elevated blood pressure is defined as systolic blood pressure $>140 \mathrm{mmHg}$ and/or diastolic blood pressure $>90 \mathrm{mmHg}$.

Method: We studied 100 patients of age more than 25 years who were diagnosed as Hypertension with or without other conditions as outdoor and indoor of Hospital.

Result: Incidence Of HTN Increased With Age.

1. Incidence of HTN is higher in men (51\%) than in female (49\%).

2. Among 100 patients $65 \%$ of patients having high SUA level.

3. In stage 1 HTN out of 28 patients, 21 patients have high SUA level, while in stage 2 HTN out of 72 patients, 44 patients have high SUA level.

Conclusion: With the result based on the study carried out we concluded that there can be a direct relation
\end{abstract} between hyperuricemia and hypertension

Key Words: hypertension, Hyperurecemia

\section{Introduction}

HTN is a sustained elevation of systemic arterial pressure. Elevated blood pressure is defined as systolic blood pressure $>140 \mathrm{mmHg}$ and/or diastolic blood pressure $>90 \mathrm{mmHg}$. In the primary (essential) hypertension no cause can be established. Recently, elevation of serum uric acid has been found to be associated with subsequent morbidity and mortality in the general population among patients with congestive heart failure, diabetics and hypertensive patients. Overproduction of uric acid and extreme hyperuricemia may also lead to a rapidly progressive form of renal insufficiency. Patients with less severe but more prolonged form of hyperuricemia are predisposed to a more chronic tubulointerstitial disorder, often referred to as gouty nephropathy. Hyperuricemia leading to hyperuric aciduria may also result in increased prevalence of nephrolithiasis.

\section{Aims And Objectives}

1. To assess serum uric acid levels in Hypertensive patients.

2. To associate changes in serum uric acid with clinical prognosis.

3. To study relation between severity of Hypertension to serum uric acid level.

\section{Review Of Literature}

The clinical features, pathogenesis, diagnosis and treatment of HTN has been well understood since long. Recently, new bio-markers for diagnosis and prognosis of HTN are under development. In recent studies, Serum uric acid has been proposed as a risk factor for hypertension and it also considered as a prognostic marker for short term prognosis.

\section{Classification of Blood Pressure}

Based on the seventh report of the Joint National Committee on prevention, detection, evaluation and treatment of high blood pressure (JNC 7 report) BP is classified into the following stages -

\begin{tabular}{|l|l|l|}
\hline $\begin{array}{l}\text { Classification Of BP For } \\
\text { Adults }>\mathbf{1 8} \text { years OLD } \\
\text { (Table-i) } \\
\text { Classification of BP }\end{array}$ & Systolic BP $\mathbf{~ m m ~ h g}$ & Diastolic BP $\mathbf{~ m m ~ h g}$ \\
\hline Normal & $<120$ & $<80$ \\
\hline Pre-hypertension & $120-139$ & $80-89$ \\
\hline Stage 1 hypertension & $140-159$ & $90-00$ \\
\hline Stage 2 hypertension & $\geq 160$ & $\geq 100$ \\
\hline
\end{tabular}




\section{Symptoms and Signs}

Uncomplicated hypertension is almost always asymptomatic, so that patient may be unaware of the consequent progressive cardiovascular damage for as long as 10 to 20 years.

Symptoms often attributed to hypertension-Headache, tinnitus, dizziness and fainting - may be observed just as commonly in the normotensive population.

When symptoms do bring the patient to the Physician, they fall into three categories. They are related to (1) the elevated pressure itself (2) the hypertensive vascular disease and (3) the underlying disease, in the case of secondary hypertension.

\section{Serum uric acid and hypertension}

Hypertension and increased SUA additionally complicate the controversies of uric acid role in cardiovascular events and the factors involved in pathogenesis of atherosclerotic consequences

In animal models it has been shown thatinduced mild hyperuricemia may contribute to endothelial dysfunction and reduction of nitric oxide (NO) levels ${ }^{69}$. Uric acid cause hypertension in rat model through the activation of the renin-angiotensin system, down regulation of nitric oxide, and induction of endothelial dysfunction and vascular smooth muscle proliferation ${ }^{64}$. It is well known how the impaired kidney function is involved in mechanisms of hypertension. The elimination of urate also depends on kidney function.

\section{Study population}

\section{Materials And Methods}

- We studied 100 patients of age more than 25 years who were diagnosed as Hypertension with or without other conditions asoutdoor and indoor of SMIMER, Hospital - Surat during year2015.

- Some of them are not on treatment, and rest are on regular treatment.

- Serum uric acid level of all patients were measured.

\section{Inclusion criteria}

A. All patients of Hypertension with or without other conditions, with on or off treatment.

B. Age group 25-85 years.

\section{Exclusion criteria}

A. Patients with gout

B. Patients is on uricosuric drug

C. Patients on drugs which increase serum uric acid level e.g salicylates ( $>2 \mathrm{gm} /$ day), diuretics, ethambutol, pyrazinamide, etc other than uricosuricdrug.

\section{a. Observation}

Here we tabulated the various observation of our study below.

\section{Age Distribution In The Study Group}

\begin{tabular}{|l|l|l|l|}
\hline Sr. no. & Age group & No. of patients & Percentage \\
\hline 1 & $25-40$ YEARS & 15 & $15 \%$ \\
\hline 2 & $41-55$ YEARS & 45 & $45 \%$ \\
\hline 3 & $56-70$ YEARS & 31 & $31 \%$ \\
\hline 4 & $\geq 71$ YEARS & 09 & $09 \%$ \\
\hline Total & & 100 & $100 \%$ \\
\hline
\end{tabular}

\section{AGE DISTRIBUTION}

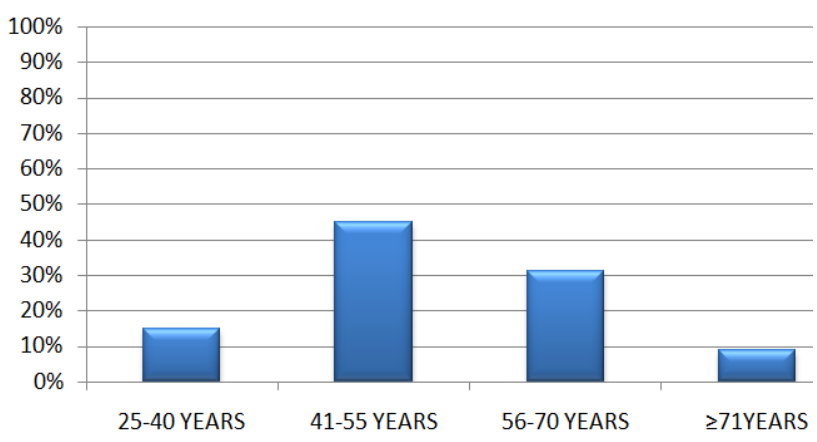


2. Sex Distribution In The Study Group

\begin{tabular}{|l|l|l|l|}
\hline Sr. No. & Gender & No. of patients & Percentage \\
\hline 1 & MALE & 51 & $51 \%$ \\
\hline 2 & FEMALE & 49 & $49 \%$ \\
\hline Total & & 100 & $100 \%$ \\
\hline
\end{tabular}

FIGURE NO. 2 SEX DISTRIBUTION

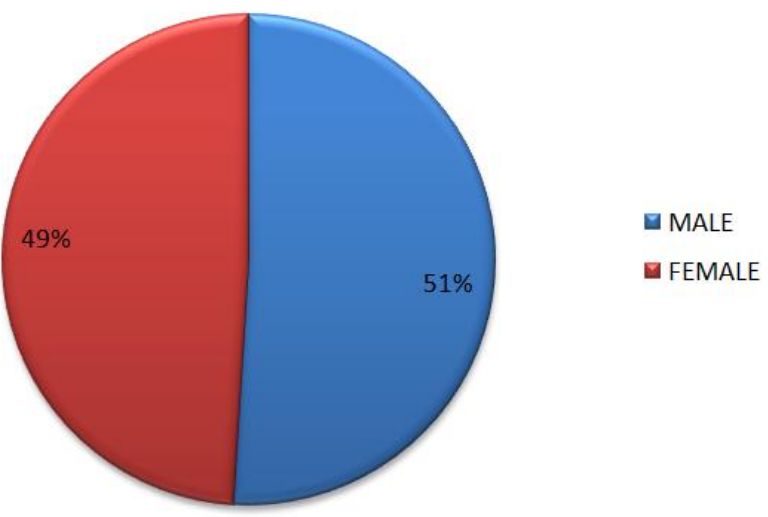

4. Evaluation Of Serum Uric Acid Levels In Htn.

\begin{tabular}{|l|l|l|}
\hline Result & No. of patients & Percentage \\
\hline Normal & 35 & $35 \%$ \\
\hline Increased & 65 & $65 \%$ \\
\hline
\end{tabular}

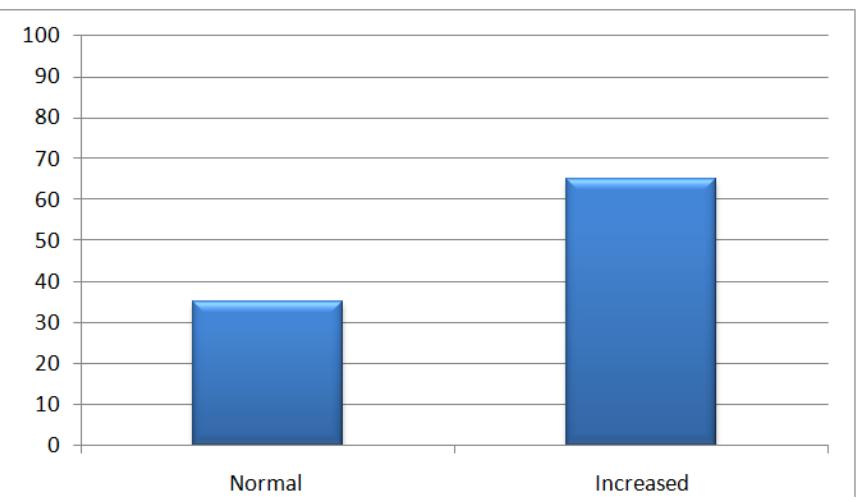

$35 \%$ of patients included in this study had normal serum uric acid level, while $65 \%$ of patients had high serum uric acid level.

5. Serum Uric Acid Level Based On Stage Of Htn (Jnc 7)

\begin{tabular}{|l|l|}
\hline Stage of HTN & No. of patients \\
\hline Stage 1 & 28 \\
\hline Stage 2 & 72 \\
\hline
\end{tabular}

\begin{tabular}{|l|l|l|l|}
\hline Stage of HTN & SUA level & No. of patients & Percentage (\%) \\
\hline Stage 1 & Increased & 21 & $75 \%$ \\
\hline & Normal & 07 & $25 \%$ \\
\hline Stage 2 & Increased & 44 & $61.111 \%$ \\
\hline & Normal & 28 & $38.889 \%$ \\
\hline
\end{tabular}




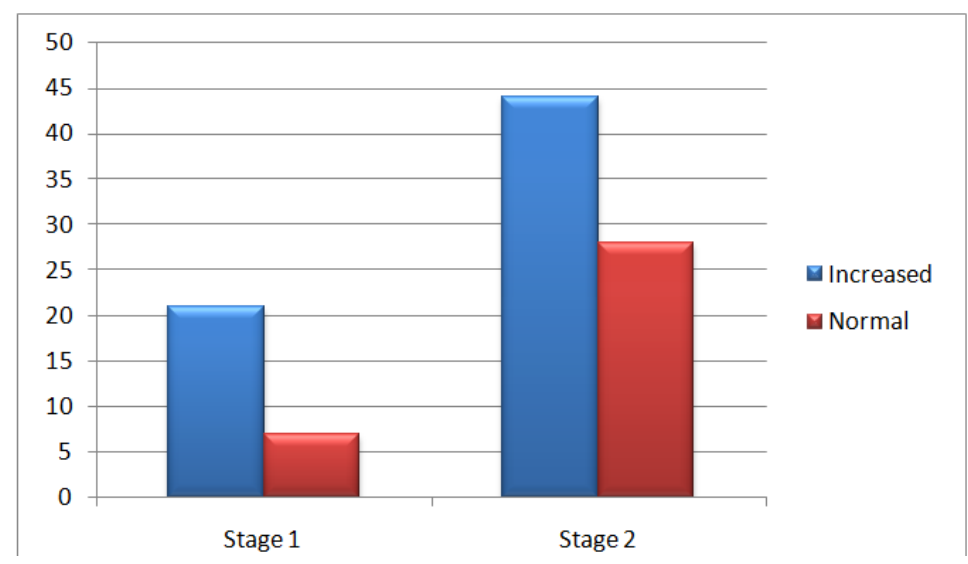

In this study 28 number of patient are in stage $1 \mathrm{HTN}$ out of which 21 number $(75 \%)$ of patients having elevated SUA level, while 72 number of patients are in stage 2 HTN out of which 44 numberof $(6.1 . \%)$ of patients having elevated SUA level.

\section{Discussion}

Elevated SUA levels have been associated with an increased risk for cardiovascular disease. The potential mechanisms by which SUA maydirectly affect cardiovascular risk include enhanced platelet aggregation andinflammatory activation of the endothelium ${ }^{42}$.

In few studies, the association of SUA with cardiovascular disease was uncertain after multivariate adjustment as in the Framingham Heart Study (1985) and the ARIC study (1996), but in others the association remained certain and significant.

Various other studies have also shown that increased SUA levels were seen in hypertensive patients. Kinsey (1961) in his study with 400hypertensive patients reported a $46 \%$ incidence of hyperuricemia inhypertensives $^{43}$. Kolbe (1965) in his study of 46 hypertensive patients found 26 to be having increased SUA levels

In our study we found that there is definite relation in SUA levels between hypertensive patients and normotensive patients and there is a directly proportional relation in the levels of SUA in relation to the duration and severity of hypertension. Hence the possibility of serum uric acid acting by the production of free radicals and causing oxidative stress leading to hypertension and whether the duration and severity of hypertension lead to renal dysfunction in the form of nephrosclerosis leading to higher levels of serum uric acid has to be considered as various other studies have also show to have a positive relation in the SUA levels and hypertension.

\section{Summary}

\section{Summary And Conclusion}

Serum uric acid levels were measured in 100 patients both outdoor and indoor attending SMIMER, Hospital Surat.

1. Incidence of HTN increased with age. The maximum number of patients in this study are 41-55 years.

2. Incidence of HTN is higher in men $(51 \%)$ than in female $(49 \%)$.

3. Smoking, alcoholism, sedentary life style, obesity were major modifiable risk factor present in $35 \%, 30 \%$, $40 \%$ and $65 \%$ respectively.

4. Among 100 patients $65 \%$ of patients having high SUA level.

5. Among 100 patients 72 number of patients are in stage $2 \mathrm{HTN}$ while 28 are in stage $1 \mathrm{HIN}$.

6. In stage $1 \mathrm{HTN}$ out of 28 patients, 21 patients have high SUA level, while in stage 2 HTN out of 72 patients, 44 patients have high SUA level.

\section{VII.Conclusion}

With the result based on the study carried out we concluded that there can be a direct relation between hyperuricemia and hypertension.

Also the study showed that the SUA levels were significantly increased in patient with stage 2 hypertension in comparison with those with stage 1 hypertension, showing that the severity of hypertension also related to the SUA levels. 


\section{Bibliography}

[1]. A. Breckenridge "Hypertension and Hyperuricemia" The Lancet 1966:287; 15-18

[2]. $\quad$ Frohlich ED “Uric acid: A risk factor for coronary heart disease." JAMA1993; 270:378-379.

[3]. World Health report 2002. Reducing Risks and Promoting Healthy Life Geneva, Switzerland: World Health Organization; 2002: 7 , 58. http ://www.who.int/whr/2002.

[4]. Vasan R S, Beiser A, Sheshadri S, et al. "Residual lifetime risk for developing hypertension in middle-aged women and men: The Framingham Heart Study." JAMA 2002; 287:1003-1010.

[5]. Lewington S, Clerk R, Qizilbash N, et al. "A meta-analysis of individual data for 1 million adults in 61 prospective studies". Prospective Studies Collaboration. Lancet 2002:360:1903-1913.

[6]. SHEP Cooperative Research Group. "Prevention of Stroke by antihypertensive drug treatment in older patients with isolated systolic hypertension, Final results of the Systolic Hypertension in the Elderly Program (SHEP)". JAMA 1991; 265:3255-3264.

[7]. Staessen JA, Thijis L, Fagard R et al. "Predicting cardiovascular risk using conventional vs. ambulatory blood pressure in older patients with systolic hypertension". JAMA 1999; 282:539-546.

[8]. Norman M Kaplan, Braunwald's textbook of Cardiovascular Medicine $7^{\text {th }}$ edition, "Systemic Hypertension: Mechanism and Diagnosis"; Elsevier Saunders 37:962.

[9]. Aram V. Chobanian, George L. Bakris, et al "The Seventh Report of theJoint National Committee on Prevention, Detection, Evaluation, andTreatment of High Blood Pressure" JAMA,2003, May 21,vol 289; 256 1-2562. 